CORE SKILLS

\title{
PAIN MANAGEMENT
}

\section{INTRODUCTION}

Acute pain (pain) is a common complaint in the pediatric inpatient setting and is most often associated with exacerbations of chronic diseases, trauma, burns or surgical and diagnostic procedures. Children with acute pain may also have chronic pain due to an underlying illness or previous injury. Chronic pain complicates effective control of acute pain and may be associated with neuropsychological changes that impact pain perception. Despite advances in understanding of the pathophysiology and management of pain in children, several barriers to effective pain management exist, such as fear of harmful side effects and drug dependency. Pediatric hospitalists should enhance pain management services through the direct provision of effective care, and are often in the best position to lead development of a systematic approach to pain management in institutions and communities.

\section{KNOWLEDGE}

Pediatric hospitalists should be able to:

- Describe the pathophysiology and multidimensional aspects of pain in children of various ages.

- Explain how pain, anxiety, and fear interrelate and discuss strategies for addressing each.

- List the indications and contraindications for the main classes of drugs used for pain management, such as opioids, non-steroidal anti-inflammatory drugs, and topical and local anesthetics.

- Discuss the pharmacology of medications commonly used for analgesia, including route of administration, dosing range, and expected side effects.

- Discuss the pharmacology of medications used for anxiolysis, including route of administration, dosing range, and expected side effects.

- Describe the effects of age, anatomy, and disease process on the pharmacology of medications used for analgesia and anxiolysis.

- Compare and contrast the risks and benefits of various modalities of drug delivery attending to drug delivery, side effects, and invasiveness and safety of delivery methods/devices.

- List appropriate monitoring techniques for patients receiving analgesics, anxiolytics, and other associated medications.

- Describe the pharmacology of and indications for reversal agents for specific classes of drugs used for pain management.

- Discuss how use of adjuvant medications, such as antidepressants, anticonvulsants, anxiolytics, and sleep medications can be used most appropriately for pain management.

- Discuss how complementary techniques such as behavioral therapy, play therapy, and physical therapy can be utilized to manage pain and anxiety.

- Describe the role of the pediatric pain consultant/pain management team and discuss barriers to local availability.

\section{SKILLS}

Pediatric hospitalists should be able to:

- Accurately assess the presence and level of pain in children regardless of developmental level utilizing history, physical examination, physiologic parameters, and validated pediatric pain scales.

- Appropriately prescribe doses of analgesic medication that ameliorate pain while avoiding untoward side effects.

- Demonstrate proficiency in adjusting drug doses in the face of breakthrough pain.

- Safely prescribe equi-analgesic doses or adjust doses appropriately when changing from intravenous to oral therapy or when switching from one medication to another.

- Select and order pain and anxiety medications in safe and cost-effective manner.

- Correctly calculate and order a pain and anxiolytic medication tapering regimen that avoids withdrawal symptoms or breakthrough pain.

- Perform careful reassessments daily and as needed, note changes in clinical status, pain, side effects, and withdrawal symptoms and respond with appropriate actions.

- Order appropriate monitoring and correctly interpret monitor data. 
- Anticipate and recognize potential side effects of analgesic and anxiolytic medications and respond with appropriate actions.

- Consistently utilize non-pharmacologic methods as part of a pain management plan.

- Identify patients likely to have chronic pain, and involve appropriate consultants to assist with long term management.

- Identify patients with neuropathic pain and develop a treatment plan with assistance from appropriate consultants.

- Correctly identify discharge needs and create a comprehensive discharge plan attending to equipment, medications, and specialty services required.

\section{ATTITUDES}

Pediatric hospitalists should be able to:

- Educate patients and the family/caregiver on various aspects of pain, including etiologies, management, and impact on the healing process.

- Involve the primary care provider in the therapeutic process early in the hospitalization and work together to coordinate appropriate follow-up care.

- Recognize the impact of uncontrolled pain has on patients' emotional and physical well-being.

- Collaborate with subspecialists and the primary care provider to ensure coordinated longitudinal care for children receiving chronic pain management services.

\section{SYSTEMS ORGANIZATION AND IMPROVEMENT}

In order to improve efficiency and quality within their organizations, pediatric hospitalists should:

- Work with hospital administration, hospital staff, subspecialists and others to implement a comprehensive, systematic approach to pain management across the continuum of care.

- Lead, coordinate or participate in the development and implementation of cost-effective, safe, evidence-based care to standardize the evaluation and management for hospitalized children with pain.

- Educate other healthcare providers who may work with children on pediatric pain assessment and safe medication use.

- Work in consultation with surgical staff to prioritize and improve the management of pain in pediatric surgical patients. 